Meta

Journal des traducteurs

Translators' Journal

\title{
Sociologie des discours scientifiques. Quelques réflexions
}

\section{Ad Hermans}

Volume 40, numéro 2, juin 1995

Usages sociaux des termes : théories et terrains

URI : https://id.erudit.org/iderudit/003065ar

DOI : https://doi.org/10.7202/003065ar

Aller au sommaire du numéro

Éditeur(s)

Les Presses de l'Université de Montréal

ISSN

0026-0452 (imprimé)

1492-1421 (numérique)

Découvrir la revue

Citer cet article

Hermans, A. (1995). Sociologie des discours scientifiques. Quelques réflexions. Meta, 40(2), 224-228. https://doi.org/10.7202/003065ar

\section{Résumé de l'article}

Les sciences constituent des réalités sociales sui generis, des systèmes structurés d'idées et de pratiques, obéissant en premier lieu à un ensemble de lois qui leur sont propres : la scientificité. Cette scientificité ne s'explique que secondairement par des variables sociales. Le discours scientifique est une des instances de l'entreprise scientifique. Il fonctionne, à son tour, d'une maniere indépendante du conceptuel et du technique. Un des objectifs de la terminologie est de décrire la dynamique des termes dans le discours et celle du discours dans l'entreprise scientifique. L'utilité de cette perspective est illustrée par une ébauche d'analyse des publications scientifiques destinées aux pairs.
Ce document est protégé par la loi sur le droit d'auteur. L'utilisation des services d'Érudit (y compris la reproduction) est assujettie à sa politique d'utilisation que vous pouvez consulter en ligne.

https://apropos.erudit.org/fr/usagers/politique-dutilisation/ 


\title{
SOCIOLOGIE DES DISCOURS SCIENTIFIQUES. QUELQUES RÉFLEXIONS
}

AD HERMANS

Instrur L.hre Morre' Haps. Brunclle's, Belgique

\begin{abstract}
Résumé
Les sciences constituent des réalités sociales sui generis, des systemes structurés

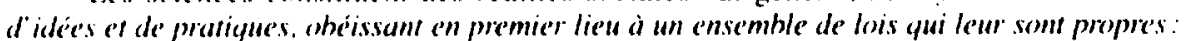
la scientificité.

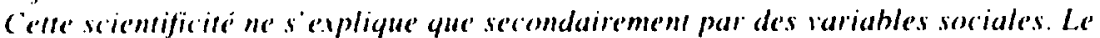

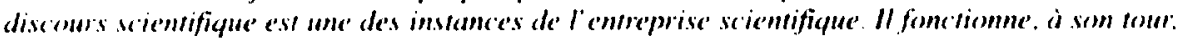

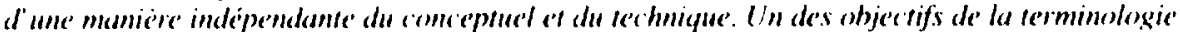

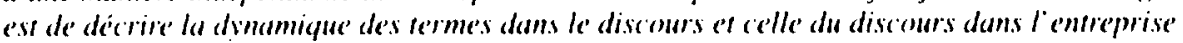
sciemificulice

L'uritite de cette perspective e'st illustré par une chanche d'analyse des publications screntificuses destimess aus pairs
\end{abstract}

La question qui a guidé la rédaction de ces quelques réflexions peut être formulée ainsi: en quoi un point de vue sociologique peut-il être utile à une perspective terminologique, telle qu elle est élaborée actuellement par des linguistes ou des sociolinguistes? Quels types de questions la sociologie peut-elle poser en matière de terminologie au sens large. donc à propos des langues de spécialité ou. selon la perspective adoptée. du discours scientifique et technique?

Nous savons que la sociologie prend ses distances par rapport aux explications psychologiques des phénomènes telles que: ale discours scientifique est hermétique parce que les scientifiques veulent écarter les non-initiés et restreindre le public auquel ils s'adressent». Dans une perspective sociologique. on se demande en effet: en quoi la volonté des scientifiques d'écarter les non-initiés par un discours hermétique. en admeltant qu'il s'agisse d'une action délibérée, constitue-1-elle (ou non) un élément de l'élaboration de l'idée de scientificité" Comment cette volonté peut-elle donc être considérée comme un élément de la méthode scientifique stricto sensusu. alors que ce fait n'est pas encore répertorié par l'épistémologice? Une perspective sociologique ccarte également les «facteurs sociaux». extra-scientifiques, comme éléments d'explication des phénomènes. Elle n’a, en effet. aucun critère pour décider ce qui est scientifique el ce qui ne l'est pas.

La question scxiologique, dans sa généralité. peut être formulée de deux manières: 1. Comment fonctionne le discours dans une science? (formulation fonctionnaliste) 2. Comment le discours scientifique se positionne-t-il dans l'univers des discours? (formulation structuraliste). La deuxième approche a été adoptée par Gusfield (1986) qui. utilisant les méthodes de Weber. compare le discours scientifique et le discours bureaucratique. Pour ma part, je poserai la question de la première façon, moins élégante. mais qui permet, dans un premier temps, de délimiter le phénomène à étudier.

\section{LA SOCIOL,OKIE DE: I A CONNAISSANCF SCIENTIFIQUF.}

Un bilan de la sociologie de la connaissance scientifique a été dressé récemment (Boudon et Clavelin 1994). Celte sociologie a une longue tradition, puisque Simmel. 
Durkheim et Weber s'y sont consacrés. et elle a connu, depuis La structure des révolutions scientifiques de Kuhn, une productivité particulière. De l'étude de ce qui se passe autour des sciences (motivations, intérêts, ambitions, pressions), en passant par l'étude des communautés scientifiques (organisation, compétition, bureaucratie), les sociologues sont arrivés à l'étude des contenus scientifiques mêmes. Ils examinent acomment dés éléments sociaux structurent les connaissances scientifiques" (Bloor 1982). Dans ce que les sociologues appellent ale programme fort» ou ala nouvelle sociologie de la connaissance». Fidée d'un noyau dur des sciences est abandonnée. Lidée centrale autour de laquelle ces recherches se conçoivent est l'impossibilité de séparer ce qui est purement et objectivement scientifique de ce qui est conditionné historiquement. Latour va jusqu’à affirmer:

Less circonstances (ce qui se trouve autour) ont généralement été considérées comme sans rapport avec la pratique de la science $[$... Nous ne disons pas simplement que lla science] est entourée, influencée, partiellement dépendante ou causée par les circonstances: nous allons jusqu à aftirmer que la science est entièrement le produit des circonstances. (Latour 1988: 25.5)

C'est dans ce cadre qu'il faut situer mes premières réflexions sur l'apport de la sociologie à la terminologie: elles faisaient dépendre le statut scientifique d'un discours du statut du rédacteur, des récepteurs et de la situation interdiscursive et considéraient le statut comme le résultat d'un jeu de reconnaissances. d'alliances, d'intérèts et d'opportunités (Hermans 1991). La science est, dans cette perspective, entièrement dissoute dans le social.

Depuis quelques années, la sociologie de la connaissance scientifique a connu un nouveau revirement. Le relativisme extrême commence à être abandonné et fait place à une nouvelle orientation. inspirée d'unce relecture de Simmel et de Durkheim et renouant avec le pragmatisme américain des années trente (Dewey 19.39). Dans celte nouvelle perspective, dont Boudon (1991) est le protagoniste principal. les sciences sont considérées comme des réalités sociales sui géneris, un monde d idées el de pratiques qui obéit en premier lieu à des lois qui lui sont propres. Une science n est pas conditionnée par la réalité sociale, mais est une réalité sociale elle-même. Less sciencess sont des institutions, au sens sociologique du terme: des manières d'agir et de penser extérieures à l'individu et qui s'imposent à lui. Les individus jouent un rôle dans la genèse des institutions. mais de la synthèse des actions individuelles se dégage une réalité spécifique. Les sciences constituent donc des réalités nouvelless. fonctionnant indépéndamment des préoccupations économiques, politiques ou de la vie quotidienne. Certains contextes sociaux et historiques favorisent une certaine vision scientifique, mais l'existence mème de celle vision ne s'explique que très secondairement par des variables sociales (Boudon 1991: 430). La scientificité est une auvre qui donne l'impression de la nécessité et de la cohérence (Boudon $1991: 431$ ) el crée ses propres valeurs et normes. Les chercheurs qui fonctionnent à l'intérieur d'une science sont influencés par des facteurs sociaux et psychologiques mais les sciences échappent pour une grande partie à celle influence.

Le fait que |la science| qui paraît constituer alu-dessus des individus quelque unité nouvelle [... | lee résout en fait] dans les actions réciproques échangées par des individus. $n$ implique pas qu'il ne laille pas reconnaitre l'existence du supra-individuel, au sens ou le sujet rencontre un état du monde. des idées, une organisation sociale qui simpose à lui (Simmel, cite par Boudon 1991).

Boudon va jusqu à remettre à l'honneur la notion de «progrès» des sciences, comme un effet collectif résultant de la pluralité des actions des scientifíques mais non poursuivi par eux. Lobjectivité scientifique est donc uné réalité suscitée par un ensemble de comportements et qui oriente ensuite ces comportements. 


\section{SOCIOIOG;E DE L.A CONNAISSANCE. ET SOCJOTERMINOLOXIF}

C'est sur le fond de celle nouvelle tendance, qui me paraît potentiellement plus féconde pour la socioterminologie que le "programme fort", que je voudrais formuler quelques réflexions. Fn effet, la socioterminologie, telle qu elle se dessine dans les travaux de Rouen. se veut une discipline utile dont le but est d'améliorer le discours scientifique. d'expliciter ce qui est implicite dans ce discours et de devenir. comme l'épistémologie positive, un moment même de l'autoconstitution des sciences. Les scientifiques ont besoin de techniciens qui conçoivent et perfectionnent leurs appareils et machines; ils ont également besoin de terminologues pour perfectionner l'instrument langagier. L'objectivité relative des sciences et la manière dont elles développent leur logique propre sont. de ce point de vue. plus intéressantes que leur conditionnement. Si la terminologie (comme discipline) existe. cést que le vocabulaire scientifique fonctionne déjà et que dans ce fonctionnement se manifeste une idée régulatrice. La première tâche de la terminologie est dès lors de décrire ce fonctionnement et de mettre au jour la dynamique des termes dans le discours el celle du discours dans l'entreprise scientifique. ou encore, selon lexpression de Gaudin (1993: 295), d'uétudier le fonctionnement réel des termes, dans leur dimension interactive et discursive». Le discours scientifique n est pas une retombée des pratiques de laboratoire. ni un simple moyen de communication, mais une instance active en interaction avec d'autres instances qui constituent une science.

\section{A TITRE D'LLLUSTRATION : IES PUHLIC ATIONS DESTINEFS AUX PAIRS}

$\dot{A}$ titre d'illustration de la démarche, examinons, dans cette perspective. la place dans l'entreprise scientifique des publications destinces aux pairs (peer writing) et publiées dans des revues qui font autorité dans la communauté scientifique (gate keepers). Selon Latour (1988: 69) ces publications constituent $55 \%$ des écrits scientifiques, et les communautés scientifiques les plus diverses leur accordent un rôle très important. Elles font partie, au même titre que les laboratoires, de la science qui se constitue, contrairement aux manuels ou à la littérature de vulgarisattion, qui présentent la science constituée. La sociologie sefforcera d'examiner comment ces publications fonctionnent à l'intérieur de l'entreprise scientifique. On admet donc que ce discours est effectivement régi par des règles. et pas seulement par des intérêts et dès stratégiess.

Ces publications constituent une logique reconstruite. Cette assertion est déjà ancienne et a ćté remise en doute à plusieurs reprises par les épistémologues, mais elle est toujours utile pour une approche sociologique. Les publications ne consignent pas ce que le chercheur a réellement fait ou ce qui s"est réellement passé dans le laboratoire. Elles ne sont pas, en premier lieu, un transfert d'informations. Elles reconstruisent. selon une certaine logique. dont il convient d'examiner les règles el les contraintes, un ensemble de pratiques qui se sont déroulées selon une autre logique. Filles sont un effort pour rendre intelligibles ces pratiques el ces événements. Kaplan (1964) fait la comparaison avec le récit des rêves. Ce n'est pas ce que le rêveur a réellement rêvé qu il raconte. mais quelque chose de plus intelligible. (ette logique reconstruite est une idéalisation. une rationalisation. dans laquelle des considérations d'ordre pratique ne trouvent pas leur place (opportunités. budgets, pressions politiques, intérêts des chercheurs, raisons suivant lesquelles certaines recherches sont effectuées, et d'autres non. et autres péripéties souvent anecdotiques).

Une sociologie des publications scientifiques s'efforcera de rendre explicites ces règles, qui sont adoptées d'une manière inconsciente par leurs auteurs, et d'examiner comment ces règles contribuent à la créalion de la ligure de la scientificité. La question posée est donc celle-ci: indépendamment du contenu particulier de la publication, quelles 
sont les conditions formelles pour que, en accord avec l'intuition des membres de la communauté scientifique. une publication soit considérée comme scientifique?

Parmi cess règles implicites, quelques-unes ont déjà été mises au jour. Pour la reconstruction. l'auteur se conforme par exemple à l'image de la scientificité telle qu elle est véhiculée par les modèles épistémologiques de son époque (Kaplan 1964). Le modèle de reconstruction le plus largement accepté a été celui de ta méthode hypothético-déductive: le scientifique arrive, par une observation minuticuse et un peu d'intuition, à formuler des hypothèses: il construit de nouvelles observations pour tester ces hypothèses, qui sont alors confirmées ou infirmées. Callon et Latour (1982) ont bien montré comment cette présentation est différente de “la science telle qu elle se fait". Mais une telle présentation reflète la métathéorie de la scientificité. la maintient et la fait également évoluer imperceptiblement.

Une deuxième caractéristique, facilement observable, des publications scientifiques est l'obéissance de l'auteur à des règles honorifiques strictes: il ne s'adresse qu'à ses pairs. Il en résulte d'une part une séparation nette entre recherche et vulgarisation et. par l'absence de toute connotation didactique, une distinction entre manuels el publications scientifiques proprement dites. Ces techniques honorifiques n'ont pas été reprises dans des modèles épistémologiquess qui, jusqu à présent, ont rélégué cés éléments au rang de facteurs sociaux ou psychologiques. Elles pourraient cependant être considérées comme partie intégrante des pratiques scientiliques mêmes. Ce trait distingue le discours scientifique d'autres discours tels que les discours religicux. politique, syndical. etc. Une des raisons pour lesquelles l'homéopathic et la parapsychologie ne sont pas promues au rang des sciences et ne sont donc pats scientifiques est qu'elles $n$ 'ont pas maitrisé cette technique et se sont adressées directement au grand public.

D'autres caractéristiques de ces publications, telles que l'effacement du sujet (l'autorité de l'auteur $n$ 'est pas charismatique ni liée à son statut, mais basée sur la rationalité de l'entreprise scientifique) (Kourilova 1993). el l'utilisation d'un vocabulaire cryptique (il suffï de disposer de la clé pour décrypter: vous n'avez pas besoin d'un augure pour le comprendre. comme cest le cas pour le vocabulaire delphique des discours quasiscientifiques) (Riggs 1986) devraient être admises au rang des lechniques scientifiques et trouver leur place dans un modèle d'analyse de l'entreprise scientifique.

\section{CONCI.USION}

À première vue. celle approche des sciences comme domaines de la réalité sociale fonctionnant d'une manière indépendante. et du fonctionnement du discours à l'intérieur de celte institution. semble s'opposer à un thème majeur de la socioterminologie. Les socioterminologues considèrent, en effet, que ale partage du savoir en domaines est une réduction idéologique» qui entrave une analyse du fonctionnement réel des discours scientifiques et techniques (Gambier 1991). Il me semble que celte opposition n'est qu'apparente. Le concept de scientificité comme institution indépendante n est qu'un concept opératoire et pratique. un point de départ ou, dans la terminologie de Weber. un type idéal: il permet. par la suite et au cours de l'analyse, de découvrir comment le fonctionnement réel de l'entreprise scientifique s'écarte du modele. Les rapports des sciences avec d’autres institutions. le pouvoir politique et l'idéologie d'une société ne sont pas niés. Mais il se peut que ces influences ne dévient qu accessoirement le mouvement de la pensée scientifique (Granger 1994), et contribuent surtout à son élaboration. 


\section{REFERENCES}

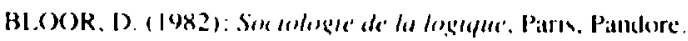

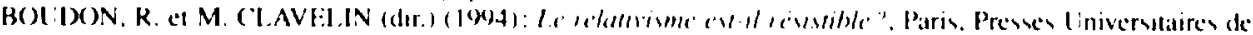
Framce.

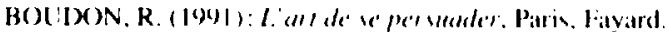

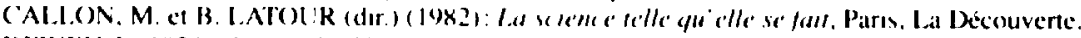

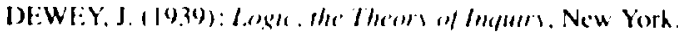

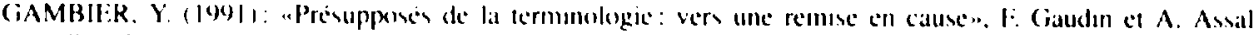

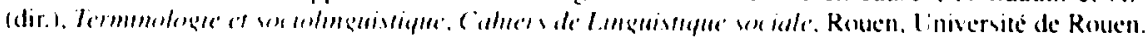

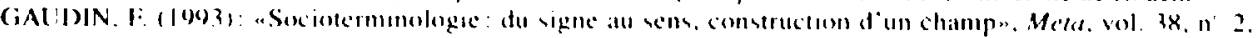

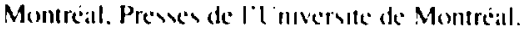

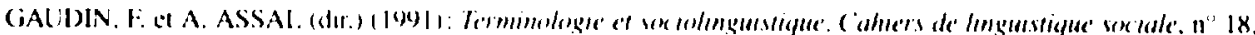

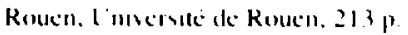

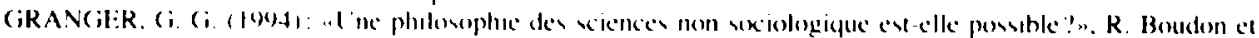

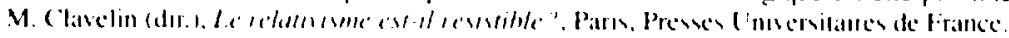

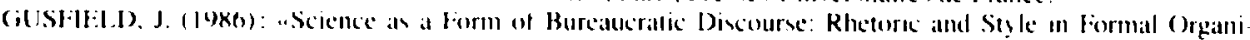

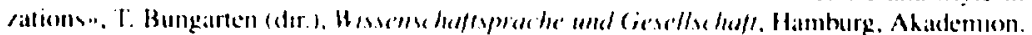

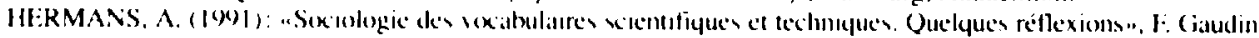

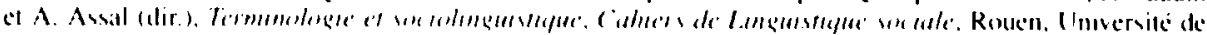
Rouen.

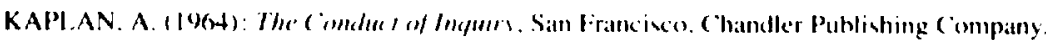

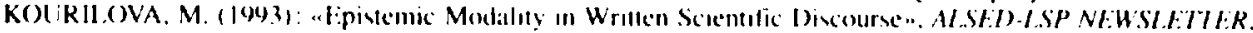
15.2 .

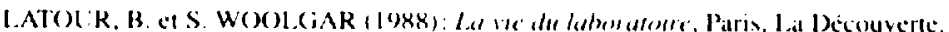

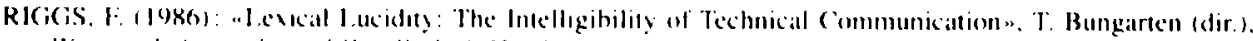

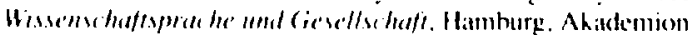

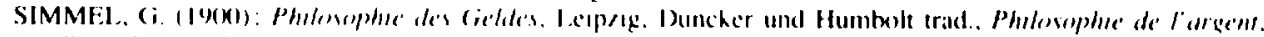

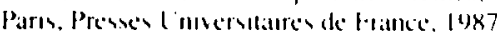

